\title{
Experiencias adversas en la infancia. Conocimiento y uso por médicos residentes de pediatría
}

\author{
Abigail Casas-Muñoz, ${ }^{1 *}$ Arturo Loredo-Abdalá, ${ }^{1}$ Betsabé Sotres-Velasco, ${ }^{2}$ Leslie V. Ramírez-Angoa, ${ }^{3}$ \\ Juan A. Román-Olmos y Giancarlo H. Cristerna-Tarrasa ${ }^{3}$ \\ ${ }^{1}$ Instituto Nacional de Pediatría, Coordinación de Estudios Avanzados sobre Maltrato Infantil-Prevención; ${ }^{2}$ Universidad Nacional Autónoma de \\ México, Facultad de Ciencias; ${ }^{3}$ Instituto Nacional de Pediatría, Dirección de Enseñanza. Ciudad de México, México
}

\section{Resumen}

Introducción: Las experiencias adversas en la infancia (EAl) se han relacionado con la adquisición de conductas de riesgo y el desarrollo de enfermedades crónicas y mentales, desde la adolescencia y en la vida adulta. Objetivo: Identificar el conocimiento y la frecuencia con la que médicos residentes de pediatría interrogan sobre las EAl. Métodos: Mediante una encuesta en línea enviada a todos los médicos residentes del año académico 2017-2018 de un hospital pediátrico de tercer nivel, se recabaron variables demográficas, del conocimiento, uso, entrenamiento y barreras para interrogar sobre EAI. Resultados: $21 \%$ de los residentes respondió la encuesta, la mayoría fue del sexo femenino (70\%), menos de $5 \%$ de los participantes estaba familiarizado con las EAl, 31 \% interrogaba sobre ellas a los padres e hijos y 71 \% consideró que tiene alguna barrera para interrogarlas. Conclusiones: Los participantes de este estudio mostraron un conocimiento limitado sobre las EAl, lo que repercutió en la frecuencia con la que preguntaban al respecto a sus pacientes y padres; al menos la mitad tuvo la percepción que identificarlas está fuera del alcance del pediatra.

PALABRAS CLAVE: Violencia. Experiencias adversas en la infancia. Maltrato infantil. Conocimiento sobre salud.

\section{Adverse childhood experiences. Knowledge and use by pediatrics residents}

\begin{abstract}
Introduction: Adverse childhood experiences (ACEs) have been associated with the acquisition of risk behaviors and development of chronic and mental diseases since adolescence and in adult life. Objective: To identify the knowledge and the frequency pediatrics residents ask about ACEs with. Methods: Through an online survey sent to all resident physicians of the 2017-2018 academic year of a tertiary care children's hospital, demographic variables, knowledge, use, training and barriers to interrogate and search for ACEs were collected. Results: $21 \%$ of residents answered the survey; the majority were women (70\%), less than $5 \%$ of participants were familiar with ACEs, $31 \%$ enquired about them in parents and their children, and $71 \%$ considered having some barrier to interrogate about them. Conclusions: Participants in this study showed limited knowledge about ACEs, which had an impact on the frequency they enquired about them with in their patients and their parents; at least half had the perception that it is beyond the reach of the pediatrician to identify them.
\end{abstract}

KEY WORDS: Violence. Adverse childhood experiences. Child abuse. Health knowledge.

Correspondencia:

*Abigail Casas-Muñoz

E-mail: abycas.md@gmail.com

0016-3813/@ 2019 Academia Nacional de Medicina de México, A.C. Publicado por Permanyer. Este es un artículo open access bajo la licencia CC BY-NC-ND (http://creativecommons.org/licenses/by-nc-nd/4.0/).

Fecha de recepción: 22-11-2019

Fecha de aceptación: 23-07-2020

A.C. Publicado por Permanyer. Este es un artículo open access bajo la licencia 


\section{Categorias de experiencias adversas en la infancia (EAI)}
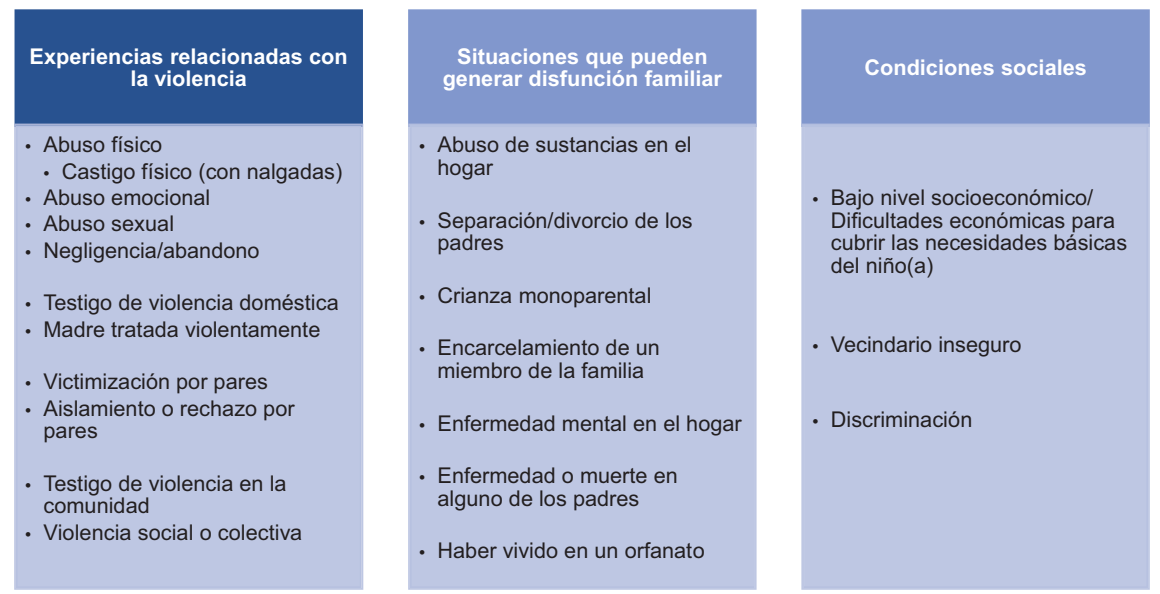

Figura 1. Categorías de experiencias adversas en la infancia ${ }^{1-4}$ (creación de los autores).

\section{Introducción}

Las experiencias adversas en la infancia (EAl) son eventos o exposición continua a circunstancias más allá del control de un niño (antes de los 18 años), que pueden afectar negativamente el bienestar de este. ${ }^{1}$ Si bien algo de estrés es normal en la vida, la activación fuerte, frecuente o prologada y la ausencia de la protección moderadora de una relación con un adulto que le brinde apoyo generan estrés tóxico (ET). Las EAl incluyen tres categorías de eventos potenciales generadores de ET (Figura 1). La acumulación de estas adversidades puede afectar la expresión de los genes, el desarrollo del sistema inmune y la respuesta al estrés.1-3

Las EAl pueden tener impacto en el desarrollo y la salud en general. Se han relacionado con la adquisición de conductas de riesgo (consumo de sustancias, fugas de casa, falta de actividad física, conductas sexuales de riesgo e ideación suicida) y el desarrollo de enfermedades crónicas y mentales (depresión) desde la adolescencia y en la vida adulta, por lo que los pediatras deben estar informados sobre su identificación y consecuencias. ${ }^{4-6}$

El objetivo de este artículo fue identificar el conocimiento y la frecuencia con la que los médicos residentes de un hospital pediátrico de tercer nivel de atención interrogan y usan en su práctica cotidiana las EAI.

\section{Métodos}

Se realizó una encuesta en línea con la herramienta www.onlineencuesta.com (versión pro). Se invitó a participar a todos los médicos residentes que estaban inscritos en las diferentes especialidades y subespecialidades que se impartieron en un hospital pediátrico de tercer nivel de atención durante el año académico 2017-2018.

Se obtuvo la autorización de la Academia Americana de Pediatría para utilizar las preguntas sobre EAI de la encuesta número $85 .^{7}$ Dos médicos bilingües realizaron dos traducciones de la encuesta y se convocó a cinco expertos en el tema, quienes las revisaron y adaptaron la mejor versión de cada pregunta para generar el instrumento final.

Se recabaron variables sociodemográficas (sexo, edad, año de residencia y universidad de procedencia) y de interés:

1. Conocimiento sobre las EAI.

2. Interrogatorio de antecedentes de EAI en los padres. Se preguntó la frecuencia con que interrogaban antecedentes de once EAl: abuso físico (AF), abuso sexual (AS), abuso emocional (AE), negligencia física (NF), negligencia emocional $(\mathrm{NE})$, escasez de alimentos, exposición a violencia doméstica (EVD), enfermedad mental o abuso de sustancias de los padres, encarcelamiento de padre, madre o cuidador y crianza monoparental.

3. Búsqueda de EAI en los niños, es decir, frecuencia con la que interrogaban sobre las seis principales EAI en sus pacientes, las cuales se agruparon de la siguiente forma:

a) Las que se interrogan habitualmente como antecedentes: padres separados o divorciados, 
enfermedad mental o abuso de sustancias en los padres.

b) Las que se preguntan por conocimiento de las EAl: encarcelamiento de padre o madre, dificultad para cubrir las necesidades básicas del niño (NF, NE, escases de alimentos) y EVD.

4. Búsqueda intencionada de maltrato infantil (MI). Se preguntó si hacían búsqueda intencionada para identificar $A F, A S, A E, N F$ y $N E$ en sus pacientes.

5. Barreras para no interrogar sobre las EAI. Se pidió que evaluaran 14 situaciones como barreras para interrogar las EAl.

6. Creencias sobre las EAI. Se preguntó el grado de acuerdo con ocho afirmaciones verdaderas relacionadas con las EAl y su asociación con el desarrollo de siete enfermedades.

7. Entrenamiento en salud mental. Se preguntó sobre rotaciones por pediatría conductual y del desarrollo, psiquiatría infantil, medicina adolescente y ciencias conductuales. Se consideró que sí en quienes rotaron más de tres semanas.

\section{Análisis estadístico}

Los datos faltantes se imputaron con el método de árboles aleatorios. Se calcularon frecuencias y medidas de tendencia central de las variables sociodemográficas. Se empleó la prueba de independencia Rao-Scott (considerada significativa con un valor de $p<0.05$ ), para definir la relación entre las variables sociodemográficas y las variables de interés. $^{8}$

Mediante regresión logística se calculó la probabilidad (RM, razón de momios) de interrogar sobre EAI y realizar la búsqueda intencionada de MI a partir del conocimiento que tuvieran al respecto. Los modelos se ajustaron conforme a las características sociodemográficas, las creencias sobre las EAl y el entrenamiento en salud mental. Para los casos con valores altos de RM (casos raros) se utilizó el método de Firth para su cálculo. ${ }^{9}$

El análisis estadístico se llevó a cabo con el programa RStudio versión 1.1.423 (RStudio Inc., 2009-2018)..$^{10}$

\section{Consideraciones éticas}

Se solicitó consentimiento a todos los participantes y se les informó del manejo y resguardo de su

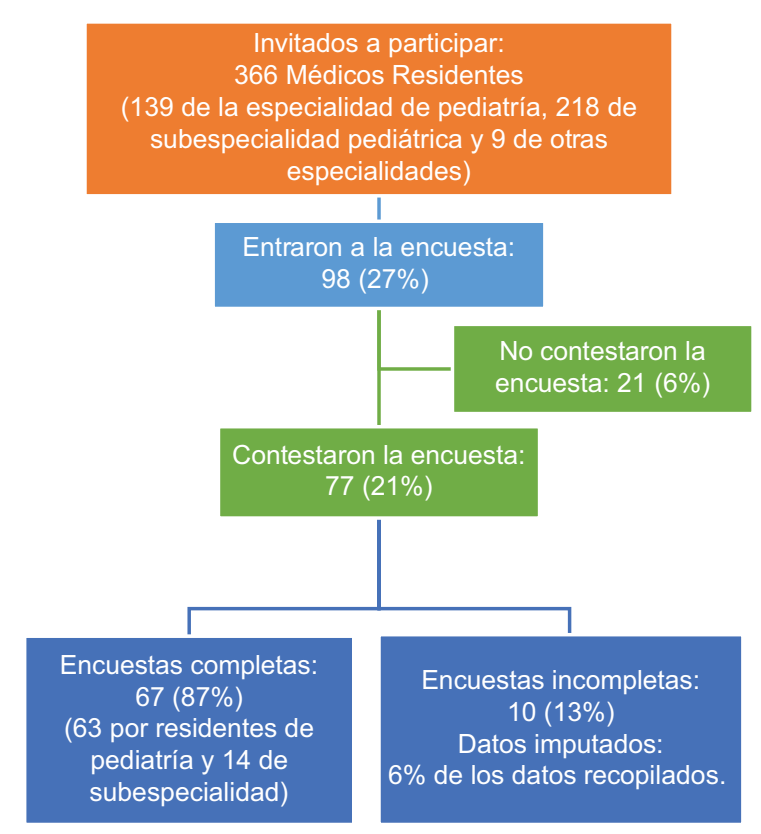

Figura 2. Diagrama de participación médicos de un hospital pediátrico de tercer nivel, de diciembre de 2017 a febrero de 2018.

información a través de un aviso de privacidad. estudio fue aprobado por los comités de investigación y de ética en investigación institucionales (número de registro 042/2017).

\section{Resultados}

Se analizó la información de 77 encuestas (Figura 2). La mayoría de los participantes fue del sexo femenino (70\%); el promedio de edad fue de 28 años (mediana de 27 años) y la mitad de los participantes (55\%) provenía de universidades de la Ciudad de México.

\section{Conocimiento sobre EAI}

El $60 \%$ de los participantes no estaba familiarizado en absoluto con el tema, $38 \%$ estaba algo o muy poco familiarizado y solo $2 \%$ se consideraba muy familiarizado. Los residentes de pediatría estuvieron más familiarizados que los de subespecialidad $(p=<0.01)$. El $18 \%$ conocía alguna EAl; las reportadas fueron AF (71\%), AE (71\%), AS (50\%), negligencia (21\%), abandono (14\%), acoso (14\%), padres alcohólicos o drogadictos (7\%), familia disfuncional (7\%), enfermedad u hospitalización prolongada de los padres $(7 \%)$ y muerte de un padre $(7 \%)$. 
Tabla 1. Búsqueda por médicos residentes, de seis experiencias adversas en la infancia en sus pacientes

\begin{tabular}{|c|c|c|c|c|}
\hline \multirow[t]{2}{*}{ Evento adverso en la infancia } & \multirow[t]{2}{*}{$\begin{array}{l}\text { Lo preguntan (\%) } \\
\qquad(\mathrm{n}=77)\end{array}$} & \multicolumn{3}{|c|}{$\begin{array}{c}\text { Diferencias por sexo de quien lo } \\
\text { pregunta }(\%)^{*}\end{array}$} \\
\hline & & $\begin{array}{l}\text { No lo } \\
\text { preguntan }\end{array}$ & $\begin{array}{l}\text { Sí lo } \\
\text { preguntan }\end{array}$ & p \\
\hline $\begin{array}{l}\text { 1. Padres separados o divorciados } \\
\text { Residente mujer (70\%) } \\
\text { Residente hombre (30\%) }\end{array}$ & 33.8 & $\begin{array}{c}70.4 \\
56.53\end{array}$ & $\begin{array}{l}29.6 \\
43.47\end{array}$ & $<0.01$ \\
\hline $\begin{array}{l}\text { 2. Padre o madre está/estuvo encarcelado } \\
\text { Residente mujer (70 \%) } \\
\text { Residente hombre (30\%) }\end{array}$ & 10.4 & $\begin{array}{l}92.6 \\
82.61\end{array}$ & $\begin{array}{c}7.4 \\
17.39\end{array}$ & $<0.01$ \\
\hline 3. Consumo de alcohol o drogas por los padres & 76.6 & & & \\
\hline 4. Madre padece o ha padecido depresión o alguna enfermedad mental & 27.3 & & & \\
\hline 5. Padres con dificultades económicas para cubrir necesidades básicas del niño & 23.4 & & & \\
\hline 6. Exposición a violencia doméstica & 15.6 & & & \\
\hline
\end{tabular}

\section{Interrogatorio sobre antecedentes de EAI en los padres}

El $74 \%$ de los residentes reportó que interrogaba sobre antecedentes de EAI en la mayoría de los padres: abuso de sustancias (30\%) o enfermedad mental $(24 \%)$ en los padres, escasez de alimentos (13\%), crianza monoparental (13\%), EVD (12\%), AE, NF y encarcelamiento de madre, padre o cuidador (4\%), AF (2\%) y AS (2\%). La cantidad de EAI que investigaban los participantes fue de una a tres (33\%), cuatro a seis $(22 \%)$ y siete a once (19\%); $26 \%$ nunca lo hacía o solo algunas veces.

\section{Búsqueda de EAl en los niños}

La mayoría de los participantes (94\%) interrogaba a sus pacientes acerca de al menos una EAI y muy pocos, las seis (4\%); una tercera parte (31\%) preguntaba sobre antecedentes de EAI tanto en los padres como en los hijos (Tabla 1). Los hombres preguntaban más sobre si los padres estaban separados 0 divorciados $(p<0.01)$ y sobre el encarcelamiento de los progenitores o cuidadores ( $<<0.01$, Tabla 1). El $88 \%$ de los participantes preguntaba acerca de los antecedentes que habitualmente se registran en la historia clínica y $43 \%$, los relacionados con conocimiento sobre EAI, principalmente los de primer año de pediatría $(p<0.05)$; el resto era menos propenso a preguntarlos $(\mathrm{RM}=0.83)$.

\section{Búsqueda intencionada de MI en los niños}

El $56 \%$ de los residentes hacía búsqueda intencionada de por lo menos una de las formas de $\mathrm{Ml}$ y el resto, casi nunca (40\%) o nunca (4\%). Se identificó que quienes interrogaban sobre las EAl en los niños también hacían dos veces más $(\mathrm{RM}=1.96)$ búsqueda intencionada de $\mathrm{Ml}$, en comparación con quienes no interrogaban a los menores $(p<0.05)$. Las formas que más frecuentemente buscaban fueron NF (42\%), AF (44\%), AE (14\%), AS (13\%) y NE (8\%).

\section{Barreras para no interrogar sobre EAl en la práctica clínica}

El $71 \%$ de los participantes consideraba que tenía barreras (Tabla 2) para interrogar sobre las EAI, de estos $5 \%$ no preguntaba antecedentes de EAI en los padres y $44 \%$ no hacía búsqueda intencionada de $\mathrm{Ml}$, proporción similar a $46 \%$ indicado por quienes no reportaron barreras. La falta de instrumentos (cuestionarios) sencillos y prácticos para evaluar el entorno familiar $(40 \%)$ fue considerada menos como barrera por las mujeres $(p<0.01$, $\mathrm{RM}=0.49$ ).

\section{Creencias de los médicos residentes sobre las EAI}

En su mayoría, los residentes de pediatría afirmaron que el ET en la niñez puede causar modificaciones 
Tabla 2. Barreras manifestadas por médicos residentes para no interrogar sobre las experiencias adversas en la infancia $(n=77)$

\begin{tabular}{|c|c|c|c|c|}
\hline Barrera & $\begin{array}{l}\text { No es una } \\
\text { barrera en } \\
\text { lo absoluto } \\
(\%)\end{array}$ & $\begin{array}{l}\text { Hasta cierto punto } \\
\text { es una barrera } \\
(\%)\end{array}$ & $\begin{array}{l}\text { Barrera } \\
\text { moderada } \\
(\%)\end{array}$ & $\begin{array}{l}\text { Barrera } \\
\text { significativa } \\
(\%)\end{array}$ \\
\hline $\begin{array}{l}\text { 1. No comprender completamente cómo la predisposición genética y las } \\
\text { experiencias tempranas en la infancia interactúan para determinar el } \\
\text { comportamiento y la salud a lo largo de la vida. }\end{array}$ & 17 & 44 & 23 & 16 \\
\hline 2. Falta de conocimiento sobre qué es el estrés tóxico. & 1 & 23 & 49 & 27 \\
\hline $\begin{array}{l}\text { 3. Falta de instrumentos (cuestionarios) sencillos y prácticos para evaluar el } \\
\text { entorno familiar en busca de factores de riesgo sociales y emocionales. }{ }^{*}\end{array}$ & 8 & 13 & 39 & 40 \\
\hline $\begin{array}{l}\text { 4. No genera beneficio detectar los factores de riesgo sociales y } \\
\text { emocionales que existen dentro de la familia. }\end{array}$ & 22 & 23 & 20 & 35 \\
\hline $\begin{array}{l}\text { 5. Los padres son renuentes a discutir los factores de riesgo sociales y } \\
\text { emocionales que existen dentro de la familia. }\end{array}$ & 3 & 26 & 29 & 42 \\
\hline $\begin{array}{l}\text { 6. Programas sociales insuficientes para abordar a las familias identificadas } \\
\text { con factores de riesgo sociales y emocionales. }\end{array}$ & 4 & 19 & 38 & 39 \\
\hline $\begin{array}{l}\text { 7. Falta de coordinación para vincular a las familias en riesgo con los } \\
\text { recursos disponibles en la comunidad. }\end{array}$ & 4 & 18 & 44 & 34 \\
\hline 8. Es incómodo preguntar a los padres sobre sus experiencias en la niñez. & 17 & 25 & 38 & 20 \\
\hline $\begin{array}{l}\text { 9. Poca disposición de los padres para hablar de sus experiencias } \\
\text { adversas en la niñez. }\end{array}$ & 3 & 18 & 44 & 35 \\
\hline $\begin{array}{l}\text { 10. Falta de entrenamiento en técnicas de crianza positivas que promuevan } \\
\text { relaciones sanas entre padres e hijos. }\end{array}$ & 4 & 17 & 48 & 31 \\
\hline $\begin{array}{l}\text { 11. Falta de material (folletos, actividades, recursos basados en la web, } \\
\text { etcétera) para promover una relación saludable de los padres con sus } \\
\text { hijos. }\end{array}$ & 8 & 29 & 38 & 25 \\
\hline $\begin{array}{l}\text { 12. No me genera beneficio aconsejar a los padres sobre una relación } \\
\text { saludable entre ellos y sus hijos. }\end{array}$ & 38 & 21 & 22 & 19 \\
\hline $\begin{array}{l}\text { 13. No tengo tiempo para aconsejar a los padres sobre una relación } \\
\text { saludable entre ellos y sus hijos. }\end{array}$ & 22 & 23 & 33 & 22 \\
\hline $\begin{array}{l}\text { 14. No tengo tiempo para detectar los factores de riesgo sociales y } \\
\text { emocionales que existen dentro de la familia. }\end{array}$ & 22 & 23 & 30 & 25 \\
\hline
\end{tabular}

*Ejemplo de factores de riesgo sociales y emocionales: depresión, abuso de sustancias de los padres, violencia doméstica, escasez de alimentos, etcétera.

epigenéticas del ADN (81.7\%), en comparación con los de subespecialidad ( $14.4 \%, p=0.05$ ). Las mujeres estuvieron menos de acuerdo en que el ET altere el desarrollo cerebral y afecte el rendimiento escolar ( $p=0.01112$,) y que los padres que experimentaron EAI significativas en la niñez tienen más dificultad para establecer relaciones estables y solidarias $(p<0.001)$.

Quienes estuvieron en desacuerdo con las aseveraciones de la Tabla 3 fueron más propensos a no hacer búsqueda intencionada de $\mathrm{Ml}$ y respecto a la asociación entre el desarrollo de enfermedades y las EAI, más de la mitad relacionó principalmente cuatro de las siete (Figura 3).

\section{Entrenamiento en salud mental}

Los participantes que tuvieron entrenamiento en salud mental realizaban más búsqueda intencionada de MI $(\mathrm{RM}=3.3844)$; destacó el $14.3 \%$ que rotó en psiquiatría infantil $(R M=6.9, p=0.017)$. Quienes rotaron por pediatría conductual, preguntaban más a los padres antecedentes de EAI en su infancia $(p=0.03112)$.

\section{Discusión}

Los resultados del presente estudio mostraron que la población participante tenía un limitado conocimiento sobre las EAI, a pesar de que solo se abordaron 
Tabla 3. Creencias de los médicos residentes de pediatría sobre el efecto de vivir experiencias adversas en la infancia y su relación con que la búsqueda intencionada de maltrato infantil

\begin{tabular}{|c|c|c|c|c|c|}
\hline \multirow[t]{2}{*}{ Creencia } & \multirow{2}{*}{$\begin{array}{c}\text { Acuerdo/ } \\
\text { desacuerdo } \\
(\%) \\
(n=77)\end{array}$} & \multicolumn{4}{|c|}{ Búsqueda intencionada de MI (\%) } \\
\hline & & $\begin{array}{l}\text { No } \\
\text { investigan } \\
(n=34)\end{array}$ & $\begin{array}{l}\text { Sí } \\
\text { investigan } \\
(n=43)\end{array}$ & $\mathbf{p}^{*}$ & RM \\
\hline $\begin{array}{l}\text { 1. El estrés fisiológico prolongado o excesivo en la niñez puede causar } \\
\text { modificaciones epigenéticas del ADN. } \\
\text { De acuerdo } \\
\text { En desacuerdo }\end{array}$ & $\begin{array}{c}96.1 \\
3.9\end{array}$ & $\begin{array}{l}41.9 \\
100\end{array}$ & $\begin{array}{c}58.1 \\
0\end{array}$ & 0.05 & 9.66 \\
\hline $\begin{array}{l}\text { 2. El estrés fisiológico prolongado o excesivo durante la niñez puede alterar el } \\
\text { desarrollo cerebral y afectar el rendimiento escolar. } \\
\text { De acuerdo } \\
\text { En desacuerdo }\end{array}$ & $\begin{array}{c}98.7 \\
1.3\end{array}$ & $\begin{array}{c}43.22 \\
100\end{array}$ & $\begin{array}{c}56.78 \\
0\end{array}$ & 0.05 & 3.89 \\
\hline $\begin{array}{l}\text { 3. Estrés fisiológico persistente en la niñez puede causar que los niños tengan } \\
\text { menos capacidad para manjar el estrés en su vida futura. } \\
\text { De acuerdo } \\
\text { En desacuerdo }\end{array}$ & $\begin{array}{c}100 \\
0\end{array}$ & $\begin{array}{c}44.15 \\
0\end{array}$ & $\begin{array}{c}55.85 \\
0\end{array}$ & - & \\
\hline $\begin{array}{l}\text { 4. Breves periodos de estrés pueden influir positivamente en el niño al } \\
\text { promover su capacidad de adaptación. } \\
\text { De acuerdo } \\
\text { En desacuerdo }\end{array}$ & $\begin{array}{c}93.5 \\
6.5\end{array}$ & $\begin{array}{c}41.67 \\
80\end{array}$ & $\begin{array}{c}58.33 \\
20\end{array}$ & 0.19 & \\
\hline $\begin{array}{l}\text { 5. La detección de factores de riesgo socioemocionales dentro de la } \\
\text { familia (por ejemplo, depresión, abuso de sustancias de los padres, violencia } \\
\text { doméstica, etc.) está más allá del alcance de la medicina pediátrica. } \\
\text { De acuerdo } \\
\text { En desacuerdo }\end{array}$ & $\begin{array}{l}53.24 \\
46.75\end{array}$ & $\begin{array}{l}48.79 \\
38.39\end{array}$ & $\begin{array}{l}51.21 \\
61.11\end{array}$ & 0.36 & \\
\hline $\begin{array}{l}\text { 6. Relaciones estables y de apoyo pueden mitigar los efectos negativos del } \\
\text { estrés persistente en la niñez. } \\
\text { De acuerdo } \\
\text { En desacuerdo }\end{array}$ & $\begin{array}{c}100 \\
0\end{array}$ & $\begin{array}{c}44.15 \\
0\end{array}$ & $\begin{array}{c}55.85 \\
0\end{array}$ & - & \\
\hline $\begin{array}{l}\text { 7.Padres que han experimentado EAl significativas en la niñez tienen más } \\
\text { dificultad para establecer relaciones estables y solidarias con sus hijos en } \\
\text { comparación con quienes no las tuvieron } \\
\text { De acuerdo } \\
\text { En desacuerdo }\end{array}$ & $\begin{array}{c}93.5 \\
6.5\end{array}$ & $\begin{array}{c}46.76 \\
60\end{array}$ & $\begin{array}{c}53.24 \\
40\end{array}$ & 0.04 & 1.98 \\
\hline $\begin{array}{l}\text { 8. El consejo del médico pediatra tiene poco efecto para mejorar las } \\
\text { habilidades de los padres en la crianza positiva de sus hijos } \\
\text { De acuerdo } \\
\text { En desacuerdo }\end{array}$ & $\begin{array}{l}51.95 \\
48.05\end{array}$ & $\begin{array}{c}40 \\
48.64\end{array}$ & $\begin{array}{c}60 \\
51.36\end{array}$ & 0.35 & \\
\hline
\end{tabular}

once $^{7-11}$ de las 19 reconocidas actualmente. Algunos participantes que tenían conocimiento nombraron otras como el acoso (bullying), la hospitalización prolongada o muerte de un familiar y la pobreza. 2,3

También se evidenció que el poco conocimiento sobre las EAl se relacionó con la baja frecuencia para interrogar sobre ellas o utilizarlas en la práctica diaria con la búsqueda intencionada de $\mathrm{Ml}$, similar a los resultados de los pediatras encuestados por la Academia Americana de Pediatría. Una causa podría ser la escasa oferta de entrenamiento sobre EAl en los currículos de medicina, ${ }^{12}$ por lo que se debe trabajar en la difusión de su conocimiento, impacto y entrenamiento, ya que los médicos se benefician no solo porque les genera conocimiento, sino habilidades y actitudes para interrogarlas. ${ }^{13} \mathrm{El}$ pobre entrenamiento sobre las EAI deriva en no poder abordar el problema, porque se teme abrir "la caja de Pandora". ${ }^{14}$ Se ha demostrado que la atención y cuidado de los médicos mejora después de haber sido capacitados sobre EAl y las respuestas sensibles que deben tener al recibir declaraciones de los pacientes..$^{15}$ Una 


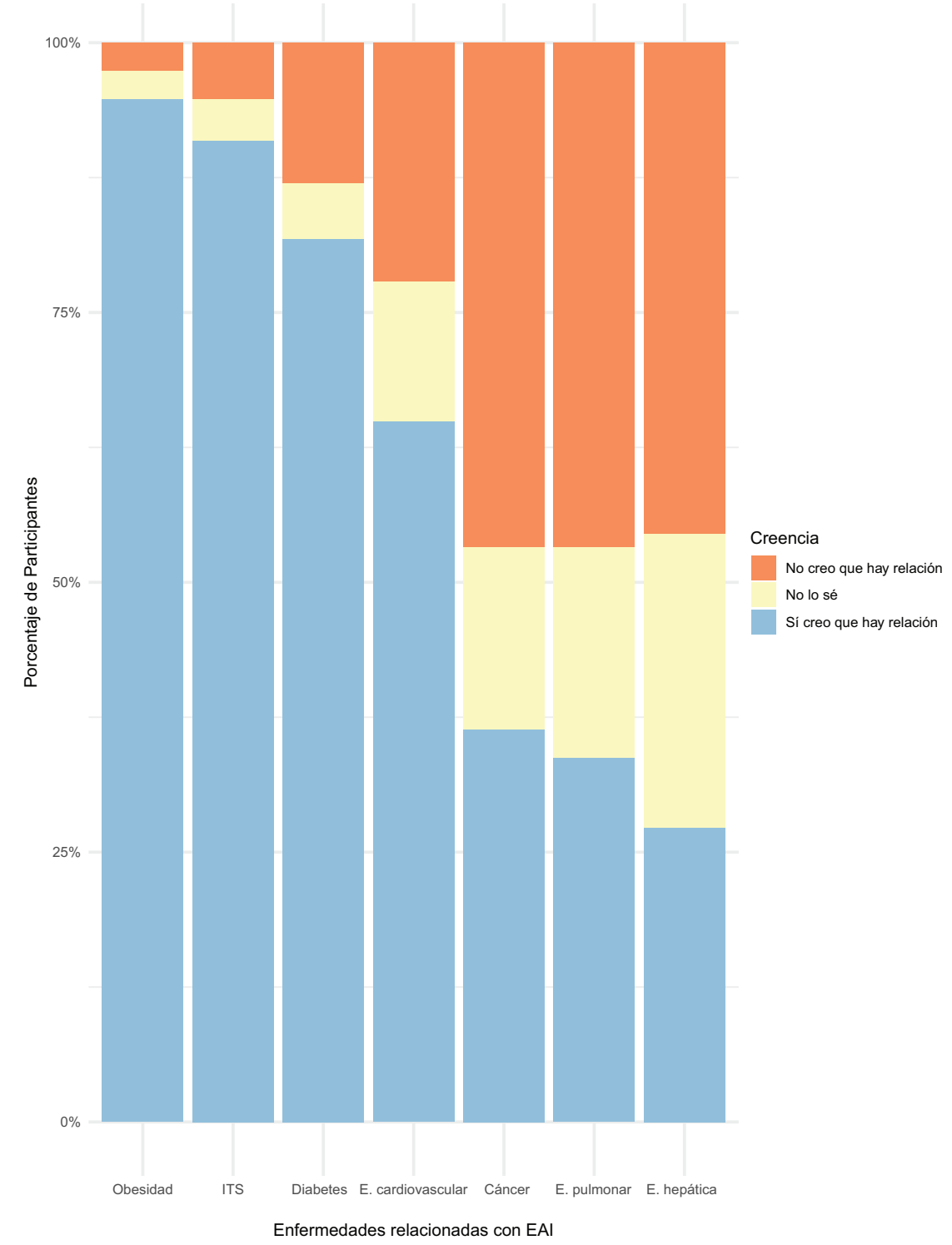

Figura 3. Creencias de los médicos residentes sobre la relación entre las experiencias adversas en la infancia (EAl) y el desarrollo de enfermedades a largo plazo. $E$ = enfermedad, ITS = infección de trasmisión sexual, EAI = experiencias adversas en la infancia.

propuesta es considerar incluir un entrenamiento sobre ellas en el plan de estudios de las universidades. ${ }^{6,16}$ Entre estas iniciativas se encuentra la asignatura optativa "Maltrato infantil: gravedad y prevención", que se imparte en el programa de Educación a Distancia de la Facultad de Medicina de la Universidad Nacional Autónoma de México. ${ }^{17}$

Otro hallazgo relevante fue la poca aplicabilidad y utilidad en la práctica clínica de los conocimientos que tenían los participantes sobre los efectos del ET en el desarrollo cerebral, la epigenética y el desarrollo de enfermedades a mediano y largo plazo. Porque, aunque los conocían, menos de la mitad interrogaba sobre las EAI que los producían y las consideran barreras o no estaban de acuerdo en el beneficio de las intervenciones y el papel que desempeña el pediatra para prevenir sus efectos, como ha pasado en otros estudios con médicos residentes. ${ }^{18}$

La mitad de los participantes tuvo la percepción o creencia que está fuera del alcance del pediatra identificar factores de riesgo socioemocionales en las familias, así como su posible intervención para mitigar sus efectos negativos. ${ }^{14}$ Tal percepción no está fundamentada, pues se ha descrito que entre 50 y $85 \%$ de los progenitores o cuidadores espera que los pediatras de sus hijos pregunten sobre estas 
situaciones. Cuando una situación grave o una preocupación familiar importante se manifiesta, los padres consideran a los pediatras como una de las primeras fuentes de ayuda para acudir. ${ }^{19}$

Al parecer, no es claro para los pediatras en formación que están en una posición privilegiada al tener en las manos un medio para transmitir e influir en la salud de un niño. ${ }^{19,20}$ Además, están en posibilidad de colaborar con trabajadores sociales, organizaciones y el Estado para que se apliquen las leyes y los cambios que se requieran. ${ }^{5,20}$ La salud y bienestar de los niños dependerá inevitablemente del estado físico, emocional y social de sus padres y de las circunstancias sociales y cuidados que tengan. La evaluación de las EAl, junto con el desarrollo y el comportamiento, podría ayudar a identificar oportunamente conductas de riesgo y a quienes están en propensión de desarrollar problemas de salud mental o enfermedades relacionadas, así como evitar su transmisión intergeneracional. ${ }^{21}$

Las principales barreras que refirieron los participantes para interrogar sobre las EAl, como en otros estudios, se relacionaron con la incomodidad de preguntar sobre estos temas, la falta de cuestionarios sencillos para identificar familias en riesgo, la falta de tiempo y la falta de conexión con servicios y programas sociales que puedan atender estas necesidades. ${ }^{22}$ Posibles soluciones a dichas barreras son las siguientes:

- Entrenamiento sobre las $\mathrm{EAl}^{23}$ y administración del tiempo de la consulta. ${ }^{14}$

- Contar con cuestionarios de fácil aplicación, situación en la que estamos trabajando actualmente para atender esta necesidad.

- Generar y dar a conocer directorios de las redes de apoyo, programas y servicios de canalización disponibles, para el aprovechamiento de los recursos comunitarios y de la sociedad civil con los que ya se cuenta. ${ }^{23}$

Respecto a las limitaciones del estudio, la tasa de respuesta fue subóptima, situación que también se presentó en la encuesta de la Academia Americana de Pediatría, ${ }^{7}$ que obtuvo un porcentaje de repuesta de $37 \%$. Esto podría relacionarse con el poco interés de los médicos por participar en encuestas enviadas a través de medios electrónicos. ${ }^{24}$ Otra de las limitantes de este estudio es el sesgo de no respuesta y que los resultados no se pueden generalizar a toda la población de residentes del Instituto, si bien brindó información valiosa sobre la situación del conocimiento y uso de los EAI para realizar sugerencias al respecto. ${ }^{24}$

\section{Agradecimientos}

Al doctor José Nicolás Reynés Manzur, director de Enseñanza, por las facilidades para realizar el estudio; así como al doctor Silvestre García de la Puente, por la asesoría metodológica.

\section{Conflictos de intereses}

Ninguno.

\section{Financiamiento}

La presente investigación recibió financiación económica del Instituto Nacional de Pediatría, a través del Fondo de Recurso Fiscal para Investigación del Programa E022-2018.

\section{Responsabilidades éticas}

Protección de personas y animales. Los autores declaran que los procedimientos seguidos se conformaron a las normas éticas del comité de experimentación humana responsable y de acuerdo con la Asociación Médica Mundial y la Declaración de Helsinki.

Confidencialidad de los datos. Los autores declaran que en este artículo no aparecen datos de pacientes.

Derecho a la privacidad y consentimiento informado. Los autores declaran que en este artículo no aparecen datos de pacientes.

\section{Bibliografía}

1. Holman DM, Ports KA, Buchanan ND, Hawkins NA, Merrick MT, Metzler $\mathrm{M}$, et al. The association between adverse childhood experiences and risk of cancer in adulthood: a systematic review of the literature. Pediatrics. 2016;138:S81-S91.

2. Finkelhor $D$, Shattuck $A$, Turner $H$, Hamby $S$. Improving the adverse childhood experiences study scale. JAMA Pediatr. 2013;167:70-75.

3. Cronholm PF, Forke CM, Wade R, Bair-Merritt MH, Davis M, Harkins-Schwarz $\mathrm{M}$, et al. Adverse childhood experiences: expanding the concept of adversity. Am J Prev Med. 2015;49:354-361.

4. Boullier M, Blair M. Adverse childhood experiences. Paediatr Child Heal (United Kingdom). 2018;28:132-137.

5. American Academy of Pediatrics. Early childhood adversity, toxic stress, and the role of the pediatrician: translating developmental science into lifelong health. Pediatrics. 2012;129:e224-e231.

6. Shonkoff JP, Committee on Psycholosocial Aspects of Child and Familiy Health, Committee on Early Childhood, Adoption, and Dependent Care A, Section on Developmental and Behavioral Pediatrics. The lifelong effects of early childhood adversity and toxic stress. Pediatrics. 2012;129:e232-e246.

7. Kerker BD, Storfer-Isser A, Szilagyi M, Stein REK, Garner AS, O'Connor KG, et al. Do pediatricians ask about adverse childhood experiences in pediatric primary care? Acad Pediatr. 2016;16(2):154-160.

8. Lipsitz SR, Fitzmaurice GM, Sinha D, Hevelone N, Giovannucci E, Hu $\mathrm{JC}$. Testing for independence in JxK contingency tables with complex sample survey data. Biometrics. 2015;71:832-840.

9. Williams R. Analyzing rare events with logistic regression. Francia: University of Notre Dame [Internet]; 2019. 
10. R: a language and envrionment for statistical computing [Internet]. Austria: R Foundation for Statistical Computing; 2019

11. Felitti VJ, Anda RF, Nordenberg D, Williamson DF, Spitz AM, Edwards V et al. Relationship of childhood abuse and household dysfunction to many of the leading causes of death in adults. The Adverse Childhood Experiences (ACE) Study. Am J Prev Med. 1992;14:245-258.

12. Pletcher BA, O'Connor M, Swift-Taylor ME, DallaPiazza M. Adverse childhood experiences: a case-based workshop introducing medical students to trauma-informed care. MedEdPORTAL. 2019;15:1-8

13. Alexander RC. Education of the physician in child abuse. Pediatr Clin North Am. 2016;37:971-988

14. Wen FK, Miller-Cribbs JE, Coon KA, Jelley MJ, Foulks-Rodríguez KA. A simulation and video-based training program to address adverse childhood experiences. Int J Psychiatry Med. 2017;52:255-264.

15. Magen E, DeLisser HM. Best practices in relational skills training for medical trainees and providers: an essential element of addressing adverse childhood experiences and promoting resilience. Acad Pediatr. 2017;17:S102-S107.

16. Bethell CD, Solloway MR, Guinosso S, Hassink S, Srivastav A, Ford D, et al. Prioritizing possibilities for child and family health: an agenda to address adverse childhood experiences and foster the social and emotional roots of well-being in pediatrics. Acad Pediatr. 2017;17:S36-S50.
17. Universidad Nacional Atónoma de México/Secretaría Sistemas Universidad Abierta y Educación a Distancia/Facultad de Medicina [Internet]. México: Licenciatura de médico cirujano; 2019.

18. Tink W, Tink JC, Turin TC, Kelly M. Adverse childhood experiences: Survey of resident practice, knowledge, and attitude. Fam Med. 2017; 49:7-13.

19. Hassink SG. Reclaiming the patient encounter. Acad Pediatr. 2017; 17:S12-S13.

20. Garner AS, Forkey H, Szilagyi M. Translating developmental science to address childhood adversity. Acad Pediatr. 2015;15:493-502

21. Marie-Mitchell A, Studer KR, O'Connor TG. How knowledge of adverse childhood experiences can help pediatricians prevent mental health problems. Fam Syst Heal. 2016;34:128-135.

22. Szilagyi M, Kerker BD, Storfer-Isser A, Stein REK, Garner A, O'Connor KG, et al. Factors associated with whether pediatricians inquire about parents' adverse childhood experiences. Acad Pediatr. 2016;16:668-675.

23. Jacob G, van den Heuvel M, Jama N, Moore AM, Ford-Jones L, Wong P. Adverse childhood experiences: basics for the paediatrician. Paediatr Child Health. 2018;24:30-37.

24. Nicholls K, Chapman K, Shaw T, Perkins A, Sullivan MM, Crutchfield S, et al. Enhancing response rates in physician surveys: the limited utility of electronic options. Health Serv Res. 2011;46:1675-1682. 